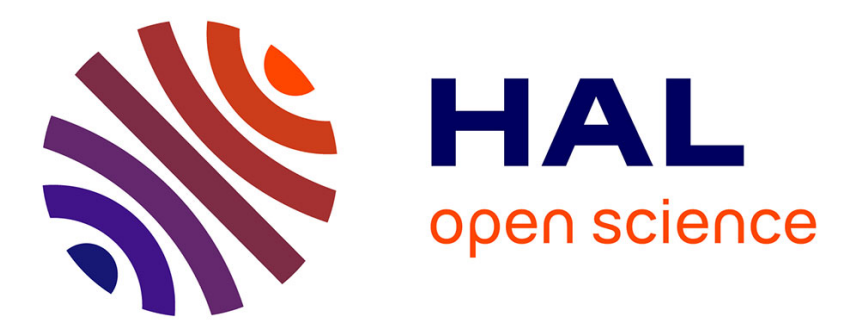

\title{
Assessment of attention demand for balance control using a Smartphone: implementation and evaluation
} Anthony Fleury, Quentin Mourcou, Céline Franco, Bruno Diot, Nicolas Vuillerme

\section{- To cite this version:}

Anthony Fleury, Quentin Mourcou, Céline Franco, Bruno Diot, Nicolas Vuillerme. Assessment of attention demand for balance control using a Smartphone: implementation and evaluation. 40th International Engineering in Medicine and Biology Conference (IEEE EMBC 2018), Jul 2018, Honolulu, United States. hal-01854793

\section{HAL Id: hal-01854793 https://hal.science/hal-01854793}

Submitted on 7 Aug 2018

HAL is a multi-disciplinary open access archive for the deposit and dissemination of scientific research documents, whether they are published or not. The documents may come from teaching and research institutions in France or abroad, or from public or private research centers.
L'archive ouverte pluridisciplinaire HAL, est destinée au dépôt et à la diffusion de documents scientifiques de niveau recherche, publiés ou non, émanant des établissements d'enseignement et de recherche français ou étrangers, des laboratoires publics ou privés. 


\title{
Assessment of attention demand for balance control using a Smartphone: implementation and evaluation
}

\author{
Anthony Fleury ${ }^{1}$ Member, IEEE, Quentin Mourcou ${ }^{1,2,3}$ Céline Franco $^{2}$, Bruno Diot ${ }^{2,4}$ and Nicolas Vuillerme ${ }^{2,5}$
}

\begin{abstract}
Dual-task paradigm studies strongly highlights the importance of considering attention demand when assessing the ability of an individual to control balance. This paper introduces the implementation of a Smartphone application for quantitative and independent assessment of attention demand for balance control. A proof-of-concept study was designed to evaluate the effectiveness of the iBalance system in assessing the attention demand for balance control. Eight young healthy adults voluntarily performed a dual-task paradigm procedure, in which they were asked to respond vocally as rapidly as possible to an unpredictable auditory stimulus while maintaining a stable seated posture and two standing postures of increasing difficulty: bipedal and unipedal. Trunk sway measurements were used as an index of postural performance, whereas reaction time measurements were used as an index of the attention demand allocated for executing the postural tasks. In line with the existing literature, results showed that, as the postural task increased in difficulty, trunk sway and attention demand used for controlling balance increased. Taken together, these results are promising, suggesting that the iBalance system could constitute a wireless, portable, lightweight, pervasive, low-cost, user-friendly Smartphone-based system for quantitative and independent assessment of attention demand for balance control suitable for home use.
\end{abstract}

Index Terms-Attention demand, Balance, Smartphone, Wearable device, Ambient Assisted Living

\section{INTRODUCTION}

A LTHOUGH it is known for decades that the control of balance involves the integration of sensory information from various sources including visual, somatosensory and vestibular systems (e.g., [1]), more recently, there has been growing interest in whether and how cognitive factors could

*This study was supported by IDS company, the French National Research Agency in the "Investissements d'Avenir" program (ANR-15-IDEX-02) of the University Grenoble Alpes, Institut Universitaire de France and IMT Lille Douai.

${ }^{1}$ Anthony Fleury (corresponding author) and Quentin Mourcou are with IMT Lille Douai, URIA, and University of Lille, F-59000 Lille, France. E-mails: anthony.fleuryeimt-lille-douai.fr

${ }^{2}$ Quentin Mourcou, Céline Franco, Bruno Diot and Nicolas Vuillerme are with Univ. Grenoble Alpes, AGEIS Laboratory, F38000 Grenoble. E-mails: celine.franco3eyahoo.fr and nicolas.vuillermeduniv-grenoble-alpes.fr

${ }^{3}$ Quentin Mourcou is with Wegoto, 38330 Montbonnot Saint-Martin. Email: quentin. mourcoulwegoto.eu

${ }^{4}$ Bruno Diot is with IDS Assistance, F-71301 Montceau-les-Mines. Email: b.dioteids-assistance.com

${ }^{5}$ Nicolas Vuillerme is with Institut Universitaire de France (IUF), F-75000 Paris. also play a role. In particular, the use of the so-called "dualtask" technique has proved extremely fruitful in understanding the attention demand of balance control.

The dual-task paradigm is a procedure in experimental (neuro)psychology that requires a participant to execute two tasks simultaneously. This technique is central to several information processing models proposing that the nervous system has a limited central capacity. It is assumed that performing a task requires a given portion of this capacity, and that if two tasks performed simultaneously require more than the total capacity, the performance of one or both tasks will be affected negatively [2], [3]. When assessing attention demand for balance control, (1) the first task, called "primary", is the one for which we want to assess the attention requirement, and usually requires the control of upright posture; (2) the second task, called "secondary", is used as an indicator of the attention demand used for the execution of the primary postural task, and usually requires a rapid verbal response to an unpredictable auditory stimulus (probe-reaction time (RT) task); (3) the performance on the secondary probe-RT task is inversely proportional to the attention demand of the primary postural task.

In general, the existing literature has reported that balance control, although mainly performed at an unconscious level, is not a fully automatic process. Indeed, even the control of a bipedal stance requires attention resources, as can be indicated by the increased RTs measured in individuals standing compared with those measured when they are sitting with support (e.g., [4], [5]). Furthermore, the amount of attention allocated to balance control has been shown to vary as a function of the complexity of the postural task being performed [4], the sensory context/environment [6], the neuromuscular constraints acting on the performer [5], and the performer's level of expertise. What is more, a common reported observation is that older adults and disabled people (e.g. [6]) dedicate more attention resources for controlling their balance than do control individuals.

These results suggest that central processing factors are an important limitation for postural control, especially in individuals showing less accurate postural capacities that could place them at a higher risk of falling and that it is very important to consider attention demand when assessing the ability of an individual to control balance. Traditionally, this assessment is mostly limited to clinical laboratory setting. 
These last years, some effort has been invested into the development of wearable technologies that could meet the requirements of independent balance testing and training at home. Along these lines, we have recently developed the socalled "iBalance" system, a wireless, portable, lightweight, pervasive, low-cost, user-friendly Smartphone-based system for quantitative and independent assessment of balance.

The structure of this paper is the following. Section II describes the iBalance system architecture. Sections III and IV present the experimental procedure and results of a proofof-concept designed to evaluate the effectiveness of the iBalance system in assessing attention demand for balance control. Section $\mathrm{V}$ discusses the results and concludes.

\section{IBALANCE SySTEM ARCHITECTURE}

The presented iBalance system [7] was developed not only to evaluate simultaneously the postural control and the associated attention demand as its predecessor but also to allow the user to design his personal balance assessment program and to enjoy it on his own without constraints of time or place. This innovating system proposes to the user an application:

(1) to quantitatively and objectively assess his balance abilities in terms of performance and attention demand;

(2) to personalize his balance training program by selftuning the system parameters based on his ability/progress/needs/preferences and/or goals and automatically launch the corresponding program for independent use;

(3) to track and display his progress history and allow the user to share it with allowed persons.

The following sections dissect the iBalance system.

\section{A. Postural performance measurement}

Trunk movements are estimated using a Kalman Filter, a standard way to compute the angles from the three different sensors (tri-axis accelerometer, magnetometer and gyroscope) that are present in the Smartphone (iPhone 4, Apple Inc.). As described in [7], a reference frame is computed as mean value of the position at the time just before the start of each trial. This allows the user to place the Smartphone wherever he wants and prevents from measurement errors that would be linked to an wrong positioning. Then, each time we sample the IMU, the current position of the Smartphone is calculated relative to this reference.

\section{B. Attention demand for balance control measurement}

During the balance assessment, the user is equipped with earphones that include a microphone to complete the auditory vocal RT task. While continuously monitoring trunk movements, the software provides unexpected, $50 \mathrm{~ms}$-long beeps at $440 \mathrm{~Hz}$ and measures the RT of the user, i.e. the time elapsed between the start of the sound and his vocalization. The elapsed time between the emission of the consecutive auditory beeps may be tuned by the user: random or uniformly distributed.

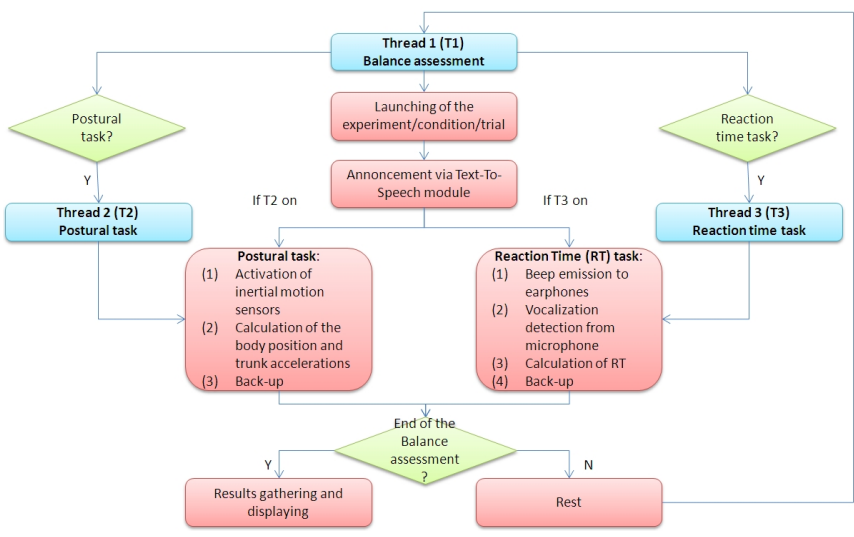

Fig. 1: iBalance software organization with the different measuring threads.

Although a common pre-processing step consists in applying a filter bank to remove most of the noise from the microphone signal, our algorithm of detection of the user's reaction was simplified at the most for computational power purposes. This signal is sampled every 10 milliseconds. At each sample, we measure the amplitude of the analog signal from the microphone denoted as $A_{m}$. From this amplitude, we estimate the power of the signal that is the integration of its square: $E(t)=\log \left(\frac{\sum_{i=1}^{t \cdot f} A_{m_{i}}^{2}}{t \cdot f}\right)$ where $f$ is the frequency and $t$ the sample number. This measure is updated every $10 \mathrm{~ms}$ and captured from half a second before the sound emission to estimate a baseline (noise present).

A strong increase of more than $15 \%$ of energy between two measures is interpreted as the beginning of the vocalization. Depending on the way the person starts speaking, a delay of one or two samples may happen. This detection step is limited to the two first seconds from the emission of a beep.

\section{C. iBalance software architecture}

The iBalance software architecture, illustrated in Figure 1, was designed to handle with three concomitant tasks. To do so, it was hinged on three different threads (allowing a better management of the memory, the computational power available on the device):

- Thread 1: Automatic management of the global balance assessment: (a) selection of postural condition, (b) trial and rest periods management and announcement by Text-To-Speech (TTS) module, (c) postural and RT data gathering and back-up, (d) feedback to the user by displaying current results in the light of his history and finally (e) sharing the complete check-up.

- Thread 2: Automatic management of the postural task: (a) computation of the reference frame, (b) data collection from the IMU, (c) calculation of body movement and trunk angulation and (d) back-up of postural data.

- Thread 3: Automatic management of the RT task: (a) sound emission to the earphones, (b) data collection from microphone and (c) RT calculation/back-up. 


\section{Automatic balance assessment}

The application is designed so that the user can train and execute the requested tasks on his own. The complete balance assessment protocol can be tuned by the user and saved into the application. To design his personalized protocol, the user may choose the balance assessment inputs: postural condition(s) (seated, unipedal and bipedal), with or without randomization, number of trial per condition, duration (in second), availability of the RT measurement, availability of the posture measurement, back-up of the recording data. If the RT measurement is on, the number of emission of auditory "beep" and their apparition in time (random or uniformly distributed) may be tuned. During the protocol, an included TTS functionality formulates the selected experimental condition 30 s before the beginning of each trial to let the user while he is taking place quietly and preparing for the test. Between two trials, few seconds are provided to the user for resting ( 45 seconds between each trials). All the resting periods and the measurement periods are described clearly to the user vocally. He is instructed of what he has to do and the system lets him enough time to adapt to the conditions required.

At the end of the balance assessment, results from both postural and cognitive tasks are gathered. Mean performances are automatically calculated for each condition and sorted. Then these results may be displayed on the Smartphone screen and possibly compared with previous sessions. They may also be communicated to outsider staff.

\section{MATERIAL AND METHODS}

\section{A. Participants}

In a proof-of-concept study, eight young healthy adults (age: $29 \pm 3$ years; height: $174 \pm 9 \mathrm{~cm}$; weight: $66 \pm 15$ $\mathrm{kg}$, mean $\pm \mathrm{SD}$ ) volunteered for this experiment. None of them presented any history of sensory and/or motor problems, neurological diseases or disorder that could affect their ability to perform the experiment. They gave their written informed consent to the experimental procedure as required by the Declaration of Helsinki and the local Ethics Committee after the nature of the study had been fully explained.

\section{B. Apparatus}

Participants wore the Smartphone mounted in a belt on the posterior low back at the level of L5 vertebra and a pair of earphones throughout the experiment [7]. The Smartphone was provided with a hands-free kit made of earphones with a piezoelectric microphone included.

\section{Task and Procedure}

We assessed the attention demand for the control of balance according to the dual-task paradigm [2]. This paradigm consists in the simultaneous execution of two concurrent tasks: (1) the first postural task, called 'primary', is the one we would like to assess the attention demand, and (2) the second cognitively demanding task, called 'secondary', is used as an indicator of the attention demand for the execution of the primary postural task. In the present experiment, the primary task required the participants to control their balance in two upright postures of increasing difficulty: (1) bipedal standing feet parallel $10 \mathrm{~cm}$ apart (Bipedal posture) and (2) unipedal standing on their dominant leg (Unipedal posture). In the Bipedal and Unipedal postures, participants were standing with eyes closed, barefoot, arms close to the trunk, and were asked to sway as little as possible.

While performing the primary postural task, participants also performed a secondary probe-RT task. The RT task required the participants to verbally respond as quickly as possible with the word 'top' to an unpredictable auditory stimulus $(50 \mathrm{~ms}, 440 \mathrm{~Hz})$. The word 'top' is used as it is unrelated to the postural task and is an easily articulated 1syllable word. For each trial, 5 auditory stimuli separated by at least $2.5 \mathrm{~s}$ were randomly presented. Number and timing of the stimuli were similar for each condition.

Participants also were submitted to a control condition in which their RT to an auditory stimulus was assessed in a seated position. Participants were sitting normally with their back supported by the backrest of the chair. No postural measures were taken as this condition (seated) only served to establish a baseline RT value for each participant. To ensure that participants did not neglect the control of upright posture in favor of attending to the auditory stimulus, the two upright postural conditions were also performed alone without executing the RT task (five "baseline" trials for each upright posture). On the whole, five 30-s trials for each experimental condition were performed. The order of presentation of the experimental conditions was randomized.

\section{Data Analysis}

Two postural and one cognitive parameters are computed:

(1) the root mean square of trunk tilt in the ML (Mediolateral) and AP (Antero-Posterior) directions (RMS in degree) as a measure of sway;

(2) the mean power frequency of trunk tilt in the ML and AP directions (MPF in $\mathrm{Hz}$ ) calculated from the power spectral density of trunk tilt: $M P F=\frac{\sum_{i=1}^{N} F F T_{i} \cdot f_{i}}{\sum_{i=1}^{N} F F T_{i}}$ where $F F T_{i}$ is the value of the Fourier Transform of the angle of the trunk in the ML and AP axis at the frequency $f_{i}$.

(3) Reaction Time (RT) score that is defined as the temporal interval between the beginning of the presentation of the auditory stimulus ("beep") and the onset of the participant's verbal responses ("top").

\section{E. Statistical Analysis}

The means of the five trials performed in each experimental condition were used for statistical analyses. Analyses of variance (ANOVAs) were used for statistical comparison of the postural conditions (using $P<0.05$ ). 


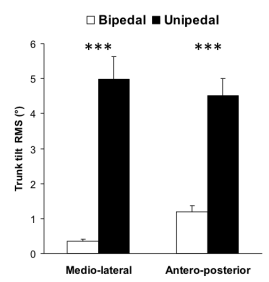

(a) Root mean square

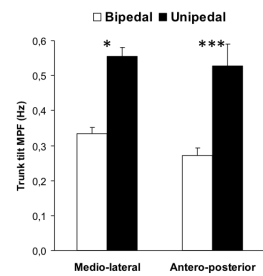
quency (b) Mean power fre-

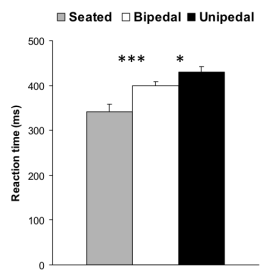

(c) Reaction time
Fig. 2: Presentation of the results for the three variables that are analyzed and for both directions of trunk tilt (Antero-posterior and Medio-lateral, considering: ***: $P<0.001$ and *: $P<0.05$.

\section{RESUlts}

\section{A. Postural data}

Analyses of the trunk tilt RMS in the ML and AP directions showed significant main effects of Posture, yielding increased values in the Unipedal posture relative to the Bipedal posture $(F(1,7)=57.18, P<0.001$, Fig. 2a, left panel and $F(1,7)=55.76, P<0.001$, Fig. 2a, right panel, for ML trunk tilt RMS and AP trunk tilt RMS, respectively).

Analyses of the trunk tilt mean MPF in the ML and AP directions showed significant main effects of Posture, yielding increased values in the Unipedal posture relative to the Bipedal posture $(F(1,7)=66.53, P<0.001$, Fig. $2 \mathrm{~b}$, left panel and $F(1,7)=10.83, P<0.05$, Fig. 2b, right panel, for ML trunk tilt MPF and AP trunk tilt MPF, resp.).

\section{B. Reaction time data}

A prerequisite of the dual-task paradigm is to check that adding the secondary task does not affect the primary. Postural data with and without RT task were compared. For Unipedal and Bipedal conditions, there was no effect of probe introduction $(P s>0.05)$. This suggests that participants did not switch attention from primary to secondary task and validates the RT data as an index of the attention requirement.

To examine the attention demand of the three postural tasks, a one-way repeated-measures ANOVA with three groups (the three postures: Seated vs Bipedal vs Unipedal) was applied to RT data. Fig. 2c illustrates the means and standard errors of mean of the RT. Results showed a main effect of Posture $(F(1,14)=24.14, P<0.001)$, yielding (1) a shorter RT in while Seated than in Bipedal and Unipedal postures $(P s<0.001)$, and (2) a longer RT in Unipedal than in Bipedal $(P<0.05)$. The goal of this statistical test is to check the influence of postural task on reaction time task and so to evaluate the attention required for the first one.

\section{DISCUSSION AND CONCLUSION}

We present a user-friendly software, on a common and affordable device to assess balance abilities and attention demand. Following dual-task paradigm, trunk sway measurement was used as an index of postural performance, whereas RT was used as an index of the attention allocated for the performance of postural tasks. By showing longer RT in upright than in seated conditions, our results first confirmed that postural control is not fully automatic but still requires a portion of the attention resources (e.g., [4], [5]). By showing longer RT in the unipedal than in the bipedal posture, our results further indicate that greater attention demand are required when the difficulty of the postural task increased [4]. Our results are in line with the existing literature. Interestingly, contrary to what it is traditionally done, the measurements of attention demand did not require the use of any expensive, dedicated and specialized equipment, neither the help of any trained experimenter/clinician. Participants were able to efficiently and independently evaluate their abilities alone, with a Smartphone.

The software guides the user in the protocol (ability to use it on his own for auto-evaluation). It does not require any other material (than the smartphone that can be owned by the patient). Using it recurrently for assessing the evolution of the parameter of a person cost nothing more. This system can be integrated in for in-home rehabilitation or prevention and the results could be stored remotely to be accessed by medical staff integrated to its medical file.

At this point, although validation studies are needed to assess whether people showing less accurate postural capacities (e.g., elderly persons), whom the consequences of an increase in attention demand for balance control could be more dramatic, are able to efficiently handle the iBalance system for independent self balance assessment, we believe that the present results are promising. Together with studies reporting positive effects of Smartphone-based Biofeedback Systems [7], [8], the present findings suggest that it could provide utility, relevance and effectiveness for assessing and training balance abilities of person at home. More largely, we believe that the iBalance system, developed as a telerehabilitation system, could represent a suitable solution for Ambient Assisted Living technologies that could facilitate the necessary shift from intra- to extra-mural care and decrease healthcare costs.

\section{REFERENCES}

[1] L. M. Nashner, F. O. Black, and C. Wall, "Adaptation to altered support and visual conditions during stance: patients with vestibular deficits," The Journal of Neuroscience, vol. 2, no. 5, pp. 536-544, 1982.

[2] D. Kahneman, Attention and effort, N. Englewood Cliffs, Ed. PrenticeHall Inc., 1973.

[3] C. Wickens, Varieties of Attention. Orlando: Academic, 1984, ch. Processing resources in attention., pp. 63-101.

[4] Y. Lajoie, N. Teasdale, C. Bard, and M. Fleury, "Attentional demands for static and dynamic equilibrium," Experimental Brain Research, vol. 97, no. 1, pp. 139-144, 1993.

[5] N. Vuillerme, N. Forestier, V. Nougier et al., "Attentional demands and postural sway: the effect of the calf muscles fatigue," Medicine and Science in Sports and Exercise, vol. 34, no. 12, pp. 1907-1912, 2002.

[6] A. Shumway-Cook and M. Woollacott, "Attentional demands and postural control: the effect of sensory context," Journals of GerontologyBiological Sciences and Medical Sciences, vol. 55, no. 1, p. 10, 2000.

[7] C. Franco, A. Fleury, P. Gumery, B. Diot, J. Demongeot, and N. Vuillerme, "iBalance-ABF: A smartphone-based audio-biofeedback balance system," IEEE Trans. Biomed. Eng., vol. 60, no. 1, pp. 211-215, 2013.

[8] B.-C. Lee, J. Kim, S. Chen, and K. H. Sienko, "Cell phone based balance trainer," Journal of NeuroEngineering and Rehabilitation, vol. 9, no. 1, p. 10, 2012. 